\title{
Economics of Areca nut Cultivation in Karnataka, a Case Study Of Shivamogga District.
}

\author{
DR. B.T. Ramappa*
}

\section{Introduction:}

The arecanut palm, Areca catechu L. is the source of the common masticatory nut,popularly known as arecanut. Arecanut is one of the most important commercial crops in the Southeast Asia. The cultivation of arecanut can be traced back to Vedic periods. Arecanut was even used in Ayurvedic and Ethane veterinary medicines. Popularly known as betel nut or supari, arecanut grown in India, China, Bangladesh, Myanmar, Thailand, Malaysia, Indonesia, Philippines \& Srilanka. India ranks first in arecanut production in the world. In India the cultivation of arecanut is mostly confined to Karnataka, Kerala \& Assam.It is also cultivated to a small extent in Tamil Nadu, West Bengal, Maharastra, Andra Pradesh, Meghalaya,Goa,Tripura, Puducherry,Mizoram,Andaman and Nicobar Islands. The share of Karnataka,Kerala \& Assam in terms of total area under cultivation and production is around 83 percent. Karnataka stands first both in terms of area \& production followed by Kerala \& Assam. The area under arecanut cultivation has increased more rapidly in Shimoga district as compared to Dakshina Kannada \& Uttara Kannada districts.

\section{USES}

1. Masticatory and Socio-Religious uses: The practice of chewing the arecanut either alone or in combination with betel leaves of pan ,lime,tobacco,camphor of spices, the combination then being called "tambula" has been in existence from time immemorial. Chewing is to increase the production of saliva and gastric juices and thus aid in the digestion.It is belived to strengthen the gums $\&$ the teeth $\&$ cleanses $\&$ deodorizes the mouth.It is also an appetizer \& a stimulant. The offering of betelnuts \& flowers,placed on a few leaves of pan, in pujas or worship is a very common,traditional time honoured practice.Persons held in esteem are offered a few pieces of arecanut with betel leaves as a sign of respect and welcome,while entering the house.Again,exchange of betelnut with betel leaves between marriage contracting parties is an important part of betrothel ceremonies throughout India.It was also a common practice for long,among the cultivating tenants in Kerala \& Karnataka \& perhaps in other states as well,to offer the landlord a few arecanuts while paying the rent.

2. Medicinal Uses: Arecanut is used against leucoderma,leprosy,cough,fits,worms,anaemia and obesity,as a purgative $\&$ as a stimulant $\&$ an appetizer

According to FAO estimation, the total area under arecanut crop in the world is 468316 hectares and the production is 593275 tonnes.Of this India's contribution in terms of area and production is 57 percent and percent 53 respectively. In terms of area and production the share of Indonesia is 16 percent \& 5 percent, China 16 percent \& 29 percent, Bangladesh 8 percent \& 5 percent, Myanmar 6 percent \& 5 percent, Thailand 2 percent each \& Malaysia 1 percent each.

The area under arecanut is around 4 lakh hectares with a production of around 4.78 lakh tons in India. Karnataka and Kerala together account for 70 percent of area and production of arecanut. In Karnataka, around 2.15 lakh hectares are under arecanut cultivation. Chickmangalore district stands first in both area and area followed by Shimoga,Davanagere districts. At present, arecanut is cultivated in 140 out of 175(80percent) of the taluks in Karnataka,with Kadur taluk ranking first in both area and production, followed by Channagiri and Bhadravathi taluks. The area under Shivamogga district arecanut is 94077.50 hectares with a production of around 52781 Metric tonns.

\section{Objectives}

- Arecanut production in the world and in India.

- Socio-economic background of sample farmers

- Problems of Arecanut Cultivators for selected area in Shivamogga District.

- To make suitable policy recommendations in the light of the analysis to influence the public policy Methodology 
* Associate Professor \& Head, Dept of Economics, Tunga Mahavidyalaya,Thirthahalli 577432 Shivamogga district ,Karnataka . ramappaeco@gmail.com

\section{Selection of the sample villages \& Farmers.}

The total sample size is 54 farmers, 19 farmers from Sagar taluk, villages were Avinahalli and E.J.Mane , 19 farmers from Thirthahalli taluk villages were Kotegadde and Hosathota and 19 farmers were selected from Gajanur \& Hossalli

Villages in Shivamogga Taluk. With in the taluk out of two villages selected for the purpose 18 farmers are selected randomly from each village equally from different farm categories and from different stages of areca garden. Accordingly, in the small farmers' category, 01 respondent in first stage, 01 -second stage \& 01 in third stages in each village (total 09) have been selected. In the medium farmers' category, 01 respondent in first stage, 01-second stage \& 01 in third stages have been selected in each village (total 09). In the large farmers' category, 01 respondent in first stage, 01 -second stage \& 01 in third stages (Total 9) have been selected in each village from the following table $1,2 \& 3$.

Key words: Arecanut,Production, Productivity,Tambula,Area,Root Grub

\section{Selection of Sample Areca Gardens}

According CAMPO Reports the life span (economic bearing) of the areca palm as 40 years the present study has divided this period into three stages.

First Stage 1 to 7 years

Second Stage 8 to 30 years

Third Stage 31 to 40 years.

In the first stage, the areca palm will be in an infant stage $\&$ it starts bearing the yield from $6^{\text {th }}$ year. The areca gardens between 8 to 30 years are considered as second stage. In this stage, the areca palms start yielding \& the yield of areca palm will go on increasing year after year upto 30 years. In the last stage may be considered as period of decline stage upto 40 years. After that the plant need to be replaced. In all 12-first stage gardens, 12-second stage gardens \& 12- third stage gardens were selected from each village.

\section{Categorization of Sample Farmers}

The respondents of the sample villages were divided into three categories as small, medium \& large based on the size of their land holdings.

Small up to 2 Acre

Medium 2 Acre to 4.00 Acres.

Large above 4.00 Acres.

Table 1Area, Production \&Yield of Arecanut in different Countries (Area: '000ha, Production

Tonnes,Productivity:KG/HA)

\begin{tabular}{|c|c|c|c|c|c|c|c|c|c|c|}
\hline \multirow{3}{*}{ Country } & \multicolumn{5}{|l|}{2001} & \multicolumn{5}{|c|}{$2009(\mathrm{P})$} \\
\hline & \multicolumn{2}{|l|}{ Area } & \multicolumn{2}{|c|}{ Production } & \multirow{2}{*}{$\begin{array}{l}\text { Produc } \\
\text { tivity } \\
\mathrm{Kg} / \mathrm{Ha}\end{array}$} & \multicolumn{2}{|c|}{ Area } & \multicolumn{2}{|c|}{ Production } & \multirow{2}{*}{$\begin{array}{l}\text { Produc } \\
\text { tivity } \\
\mathrm{Kg} / \mathrm{Ha} \\
\end{array}$} \\
\hline & '000ha, & $\begin{array}{l}\% \text { to } \\
\text { total }\end{array}$ & $\begin{array}{l}\text { '000 } \\
\text { tonne } \\
\mathrm{s}\end{array}$ & $\begin{array}{l}\% \\
\text { to } \\
\text { tota } \\
1\end{array}$ & & $\begin{array}{l}{ }^{\circ} 000 \mathrm{~h} \\
\mathrm{a},\end{array}$ & $\begin{array}{l}\% \text { to } \\
\text { total }\end{array}$ & $\begin{array}{l}{ }^{`} 000 \\
\text { tonn } \\
\text { es }\end{array}$ & $\begin{array}{l}\% \text { to } \\
\text { total }\end{array}$ & \\
\hline India & 315.20 & 52.84 & $\begin{array}{l}373 . \\
10\end{array}$ & $\begin{array}{l}52 . \\
71\end{array}$ & 1184 & $\begin{array}{l}400.0 \\
4\end{array}$ & 54.81 & 489 & $\begin{array}{l}56.3 \\
8 \\
\end{array}$ & 1222 \\
\hline Indonesia & 102.02 & 17.10 & $\begin{array}{l}45.5 \\
9\end{array}$ & $\begin{array}{l}6.4 \\
4\end{array}$ & 447 & $\begin{array}{l}125.0 \\
0\end{array}$ & 17.13 & 52 & 6.11 & 416 \\
\hline China & 51.03 & 8.55 & $\begin{array}{l}165 . \\
08\end{array}$ & $\begin{array}{l}23 . \\
32\end{array}$ & 3235 & 59.00 & 8.08 & 162 & $\begin{array}{l}18.6 \\
8 \\
\end{array}$ & 2745 \\
\hline Bangladesh & 77.80 & 13.04 & $\begin{array}{l}47.0 \\
0 \\
\end{array}$ & $\begin{array}{l}6.6 \\
4 \\
\end{array}$ & 604 & 79.00 & 10.82 & 56 & 6.46 & 709 \\
\hline Mynamar & 34.98 & 5.86 & $\begin{array}{l}51.4 \\
6 \\
\end{array}$ & $\begin{array}{l}7.2 \\
7 \\
\end{array}$ & 1471 & 36.00 & 4.93 & 57 & 6.57 & 1583 \\
\hline Thailand & 14.00 & 2.35 & $\begin{array}{l}23.0 \\
0 \\
\end{array}$ & $\begin{array}{l}3.2 \\
5 \\
\end{array}$ & 1643 & 16.00 & 2.19 & 26 & 3.51 & 1625 \\
\hline Malaysia & 1.50 & 0.25 & 2.50 & $\begin{array}{l}0.3 \\
5 \\
\end{array}$ & 740 & 0.80 & 0.11 & 1.3 & 0.15 & 1625 \\
\hline
\end{tabular}


Economics Of Arecanut Cultivation In Karnataka, A Case Study Of Shivamogga District

\begin{tabular}{|l|l|l|l|l|l|l|l|l|l|l|}
\hline Maldives & 0.05 & 0.01 & 0.04 & $\begin{array}{l}0.0 \\
1\end{array}$ & - & 0.04 & 0.01 & 0.33 & 0.04 & 8250 \\
\hline Nepal & - & & - & - & - & 2.00 & 0.27 & 3.6 & 0.42 & 2 \\
\hline Srilanka & - & & - & - & - & 12.00 & 1.64 & 20 & 2.31 & 1667 \\
\hline Keny & 0.00 & & 0.09 & $\begin{array}{l}0.0 \\
1\end{array}$ & - & - & - & 0.09 & 0.01 & - \\
\hline World & 596.50 & & $\begin{array}{l}707 . \\
80\end{array}$ & $\begin{array}{l}100 \\
.0\end{array}$ & & & $\begin{array}{l}7187 \\
8\end{array}$ & & & $\begin{array}{l}86.8 \\
2\end{array}$ \\
\hline
\end{tabular}

Source: Directorate of Arecanut and Spice Development,Calicut \& Food \& Agricultural Organisation,Rome

Table 2 Area, Production \&Yield of Arecanut in different States of Indian Union (Area: ‘000ha, Production Tonnes,Productivity:KG/HA)

\begin{tabular}{|c|c|c|c|c|c|}
\hline \multirow{3}{*}{ State } & \multicolumn{5}{|c|}{$2009-10$} \\
\hline & \multicolumn{2}{|c|}{ Area } & \multicolumn{2}{|c|}{ Production } & \multirow{2}{*}{$\begin{array}{l}\text { Productivity } \\
\mathrm{Kg} / \mathrm{Ha}\end{array}$} \\
\hline & '000ha, & $\%$ to total & $\begin{array}{l}{ }^{\circ} 000 \\
\text { tonnes }\end{array}$ & $\%$ to total & \\
\hline Andrapradesh & 0.25 & 0.06 & 0.19 & 0.04 & 754 \\
\hline Assam & 69.97 & 17.49 & 62.7 & 12.69 & 896 \\
\hline Goa & 1.85 & 0.46 & 2.78 & 0.56 & 1503 \\
\hline Karnataka & 184.52 & 21.12 & 240 & 48.58 & 1300 \\
\hline Kerala & 97.17 & 24.29 & 112.14 & 22.70 & 1154 \\
\hline Maharastra & 2.2 & 0.55 & 3.58 & 0.72 & 1626 \\
\hline Meghalaya & 12.36 & 3.09 & 17.1 & 3.46 & 1384 \\
\hline Mizoram & 6.58 & 1.64 & 8.21 & 1.7 & 1248 \\
\hline Nagaland & 0.2 & 0.05 & 1.3 & 0.26 & 6500 \\
\hline Tamilnadu & 5.03 & 1.26 & 10.39 & 2.10 & 2067 \\
\hline Tripura & 4.43 & 1.11 & 8.36 & 1.7 & 1886 \\
\hline West Bengal & 11.39 & 2.85 & 21.16 & 4.3 & 1857 \\
\hline $\begin{array}{l}\text { Andaman } \\
\text { Nicobar }\end{array}$ & 4.1 & 1.02 & 6 & 1.2 & 1463 \\
\hline Pondicherry & 0.06 & 0.01 & 0.08 & 0.02 & 13.06 \\
\hline All India & 400.12 & 100.0 & 493.98 & 100.0 & 1234 \\
\hline
\end{tabular}

Source: Directorate of Economics \& Statistics, New Delhi

Table 3 Area, Production \&Yield of Arecanut in different Districts of Karnataka (Area: '000ha, Production Tonnes)

\begin{tabular}{|c|c|c|c|c|c|c|c|c|c|c|c|c|c|}
\hline \multirow[t]{2}{*}{ District } & \multicolumn{2}{|c|}{ 1999-00 } & \multicolumn{2}{|c|}{ 2004-05 } & \multicolumn{2}{|c|}{ 2005-06 } & \multicolumn{2}{|c|}{ 2006-07 } & \multicolumn{2}{|c|}{ 2007-08 } & \multicolumn{3}{|c|}{ 2008-09 } \\
\hline & $\begin{array}{l}\text { Are } \\
\text { a }\end{array}$ & Prodn & Area & $\begin{array}{l}\text { Are } \\
\text { a }\end{array}$ & $\begin{array}{l}\text { Prod } \\
n\end{array}$ & $\begin{array}{l}\text { Are } \\
\text { a }\end{array}$ & $\begin{array}{l}\text { Prod } \\
n\end{array}$ & $\begin{array}{l}\text { Are } \\
\text { a }\end{array}$ & $\begin{array}{l}\text { Prod } \\
n\end{array}$ & $\begin{array}{l}\text { Are } \\
\text { a }\end{array}$ & $\begin{array}{l}\text { Prod } \\
n\end{array}$ & Area & $\begin{array}{l}\text { Prod } \\
n\end{array}$ \\
\hline $\begin{array}{l}\text { Bagalko } \\
\text { te }\end{array}$ & 4 & 6 & 4 & 2 & 3 & 0 & 0 & 0 & 0 & 0 & 0 & 0 & 0 \\
\hline $\begin{array}{l}\text { Bengalu } \\
\mathrm{ru}(\mathrm{U})\end{array}$ & 81 & 113 & 81 & 233 & 326 & 274 & 383 & 247 & 346 & 326 & 456 & 315 & 441 \\
\hline $\begin{array}{l}\text { Bengalu } \\
\text { ru }(\mathrm{R})\end{array}$ & 500 & 699 & 514 & $\begin{array}{l}197 \\
5\end{array}$ & $\begin{array}{l}276 \\
3\end{array}$ & $\begin{array}{l}209 \\
9\end{array}$ & 2936 & $\begin{array}{l}225 \\
7\end{array}$ & 3157 & $\begin{array}{l}137 \\
7\end{array}$ & 1926 & 1338 & 1608 \\
\hline $\begin{array}{l}\text { Belgau } \\
\mathrm{m}\end{array}$ & & & & 8 & 11 & 9 & 12 & 9 & 12 & 7 & 9 & 7 & 9 \\
\hline Bellary & 12 & 17 & 19 & 32 & 46 & 58 & 83 & 62 & 89 & 62 & 89 & 62 & 89 \\
\hline
\end{tabular}


Economics Of Arecanut Cultivation In Karnataka, A Case Study Of Shivamogga District

\begin{tabular}{|c|c|c|c|c|c|c|c|c|c|c|c|c|c|}
\hline $\begin{array}{l}\text { Chamar } \\
\text { ajanagar }\end{array}$ & 174 & 124 & 329 & 309 & 219 & 311 & 221 & 311 & 221 & 317 & 225 & 317 & 225 \\
\hline $\begin{array}{l}\text { Chikam } \\
\text { agalur }\end{array}$ & $\begin{array}{l}135 \\
06\end{array}$ & $\begin{array}{l}1547 \\
0\end{array}$ & $\begin{array}{l}141 \\
86\end{array}$ & $\begin{array}{l}166 \\
16\end{array}$ & $\begin{array}{l}190 \\
32\end{array}$ & $\begin{array}{l}181 \\
92\end{array}$ & $\begin{array}{l}2083 \\
8\end{array}$ & $\begin{array}{l}190 \\
46\end{array}$ & $\begin{array}{l}2181 \\
6\end{array}$ & $\begin{array}{l}194 \\
53\end{array}$ & $\begin{array}{l}2228 \\
2\end{array}$ & $\begin{array}{l}1975 \\
1\end{array}$ & $\begin{array}{l}2262 \\
3\end{array}$ \\
\hline $\begin{array}{l}\text { Chitradu } \\
\text { rga }\end{array}$ & $\begin{array}{l}800 \\
1\end{array}$ & 7707 & $\begin{array}{l}982 \\
7\end{array}$ & $\begin{array}{l}144 \\
98\end{array}$ & $\begin{array}{l}139 \\
65\end{array}$ & $\begin{array}{l}126 \\
88\end{array}$ & $\begin{array}{l}1222 \\
2\end{array}$ & $\begin{array}{l}148 \\
20\end{array}$ & $\begin{array}{l}1427 \\
6\end{array}$ & $\begin{array}{l}153 \\
18\end{array}$ & $\begin{array}{l}1475 \\
5\end{array}$ & $\begin{array}{l}1546 \\
5\end{array}$ & $\begin{array}{l}1489 \\
7\end{array}$ \\
\hline $\begin{array}{l}\text { Dakshin } \\
\text { a } \\
\text { Kannada }\end{array}$ & $\begin{array}{l}236 \\
02\end{array}$ & $\begin{array}{l}4210 \\
5\end{array}$ & $\begin{array}{l}254 \\
47\end{array}$ & $\begin{array}{l}272 \\
09\end{array}$ & $\begin{array}{l}485 \\
40\end{array}$ & $\begin{array}{l}273 \\
38\end{array}$ & $\begin{array}{l}4877 \\
0\end{array}$ & $\begin{array}{l}274 \\
81\end{array}$ & $\begin{array}{l}4902 \\
6\end{array}$ & $\begin{array}{l}275 \\
32\end{array}$ & $\begin{array}{l}4911 \\
7\end{array}$ & $\begin{array}{l}2757 \\
5\end{array}$ & $\begin{array}{l}4914 \\
1\end{array}$ \\
\hline $\begin{array}{l}\text { Davaneg } \\
\text { ere }\end{array}$ & $\begin{array}{l}138 \\
60\end{array}$ & $\begin{array}{l}1335 \\
1\end{array}$ & $\begin{array}{l}144 \\
76\end{array}$ & $\begin{array}{l}220 \\
15\end{array}$ & $\begin{array}{l}212 \\
06\end{array}$ & $\begin{array}{l}232 \\
41\end{array}$ & $\begin{array}{l}2238 \\
7\end{array}$ & $\begin{array}{l}227 \\
79\end{array}$ & $\begin{array}{l}2194 \\
2\end{array}$ & $\begin{array}{l}242 \\
29\end{array}$ & $\begin{array}{l}2333 \\
9\end{array}$ & $\begin{array}{l}2693 \\
1\end{array}$ & $\begin{array}{l}2594 \\
2\end{array}$ \\
\hline $\begin{array}{l}\text { Dharwa } \\
\text { d }\end{array}$ & 10 & 14 & 17 & 11 & 15 & 15 & 21 & 18 & 25 & 17 & 24 & 9 & 13 \\
\hline Gadag & & & & 3 & 4 & 3 & 4 & 3 & 4 & 3 & 4 & 3 & 4 \\
\hline Hassan & $\begin{array}{l}182 \\
5\end{array}$ & 1783 & $\begin{array}{l}192 \\
2\end{array}$ & $\begin{array}{l}348 \\
3\end{array}$ & $\begin{array}{l}340 \\
3\end{array}$ & $\begin{array}{l}368 \\
0\end{array}$ & 3596 & $\begin{array}{l}381 \\
9\end{array}$ & 3732 & $\begin{array}{l}388 \\
0\end{array}$ & 3791 & 3928 & 3838 \\
\hline Haveri & 239 & 334 & 360 & 717 & $\begin{array}{l}100 \\
2 \\
\end{array}$ & 715 & 999 & 794 & 1109 & 922 & 1288 & 1043 & 1457 \\
\hline Kodagu & $\begin{array}{l}117 \\
1\end{array}$ & 1638 & $\begin{array}{l}119 \\
8\end{array}$ & $\begin{array}{l}138 \\
6 \\
\end{array}$ & $\begin{array}{l}193 \\
9 \\
\end{array}$ & $\begin{array}{l}142 \\
3\end{array}$ & 1991 & $\begin{array}{l}229 \\
8\end{array}$ & 3215 & $\begin{array}{l}285 \\
1\end{array}$ & 3988 & 2994 & 3598 \\
\hline Kolar & 111 & 155 & 86 & 54 & 76 & 54 & 76 & 54 & 76 & 0 & 0 & 0 & 0 \\
\hline Mandya & 681 & 952 & 700 & 879 & $\begin{array}{l}122 \\
9\end{array}$ & 833 & 1164 & 868 & 1213 & 620 & 867 & 653 & 913 \\
\hline Mysore & 503 & 357 & 400 & 676 & 480 & 556 & 395 & $\begin{array}{l}102 \\
6\end{array}$ & 728 & 911 & 647 & 1109 & 787 \\
\hline Raichur & 0 & 0 & 0 & 0 & 0 & 0 & 0 & 0 & 0 & 0 & 0 & 0 & 0 \\
\hline $\begin{array}{l}\text { Ramnag } \\
\text { aram }\end{array}$ & & & & & & & & & & 868 & 1214 & 2051 & 2869 \\
\hline $\begin{array}{l}\text { Sivamog } \\
\text { ga }\end{array}$ & $\begin{array}{l}211 \\
05\end{array}$ & $\begin{array}{l}3175 \\
9\end{array}$ & $\begin{array}{l}239 \\
27 \\
\end{array}$ & $\begin{array}{l}291 \\
50\end{array}$ & $\begin{array}{l}438 \\
65 \\
\end{array}$ & $\begin{array}{l}311 \\
67 \\
\end{array}$ & $\begin{array}{l}4690 \\
0 \\
\end{array}$ & $\begin{array}{l}329 \\
38 \\
\end{array}$ & $\begin{array}{l}4956 \\
5 \\
\end{array}$ & $\begin{array}{l}354 \\
92 \\
\end{array}$ & $\begin{array}{l}5340 \\
8 \\
\end{array}$ & $\begin{array}{l}3763 \\
1\end{array}$ & $\begin{array}{l}5662 \\
7 \\
\end{array}$ \\
\hline $\begin{array}{l}\text { Tumuku } \\
\mathrm{r}\end{array}$ & $\begin{array}{l}820 \\
9 \\
\end{array}$ & $\begin{array}{l}1006 \\
9\end{array}$ & $\begin{array}{l}104 \\
28 \\
\end{array}$ & $\begin{array}{l}161 \\
97 \\
\end{array}$ & $\begin{array}{l}198 \\
67 \\
\end{array}$ & $\begin{array}{l}190 \\
44 \\
\end{array}$ & $\begin{array}{l}2336 \\
0 \\
\end{array}$ & $\begin{array}{l}195 \\
84 \\
\end{array}$ & $\begin{array}{l}2402 \\
2 \\
\end{array}$ & $\begin{array}{l}199 \\
37 \\
\end{array}$ & $\begin{array}{l}2445 \\
5 \\
\end{array}$ & $\begin{array}{l}2205 \\
8 \\
\end{array}$ & $\begin{array}{l}2705 \\
7 \\
\end{array}$ \\
\hline Udupi & $\begin{array}{l}383 \\
4 \\
\end{array}$ & 6840 & $\begin{array}{l}401 \\
2 \\
\end{array}$ & $\begin{array}{l}501 \\
9 \\
\end{array}$ & $\begin{array}{l}895 \\
4 \\
\end{array}$ & $\begin{array}{l}512 \\
7 \\
\end{array}$ & 9146 & $\begin{array}{l}517 \\
4 \\
\end{array}$ & 9230 & $\begin{array}{l}529 \\
4 \\
\end{array}$ & 9444 & 5474 & 6579 \\
\hline $\begin{array}{l}\text { Uttaraka } \\
\text { nnada }\end{array}$ & $\begin{array}{l}999 \\
8 \\
\end{array}$ & $\begin{array}{l}1359 \\
0 \\
\end{array}$ & $\begin{array}{l}111 \\
60 \\
\end{array}$ & $\begin{array}{l}122 \\
87 \\
\end{array}$ & $\begin{array}{l}167 \\
01 \\
\end{array}$ & $\begin{array}{l}143 \\
24 \\
\end{array}$ & $\begin{array}{l}1947 \\
0 \\
\end{array}$ & $\begin{array}{l}148 \\
13 \\
\end{array}$ & $\begin{array}{l}2013 \\
5 \\
\end{array}$ & $\begin{array}{l}149 \\
88 \\
\end{array}$ & $\begin{array}{l}2037 \\
3 \\
\end{array}$ & $\begin{array}{l}1580 \\
1 \\
\end{array}$ & $\begin{array}{l}1899 \\
1 \\
\end{array}$ \\
\hline Total & $\begin{array}{l}107 \\
426 \\
\end{array}$ & $\begin{array}{l}1470 \\
83 \\
\end{array}$ & $\begin{array}{l}119 \\
093 \\
\end{array}$ & $\begin{array}{l}152 \\
759 \\
\end{array}$ & $\begin{array}{l}203 \\
646 \\
\end{array}$ & $\begin{array}{l}161 \\
151\end{array}$ & $\begin{array}{l}2149 \\
74\end{array}$ & $\begin{array}{l}168 \\
401 \\
\end{array}$ & $\begin{array}{l}2239 \\
39 \\
\end{array}$ & $\begin{array}{l}174 \\
404 \\
\end{array}$ & $\begin{array}{l}2317 \\
01\end{array}$ & $\begin{array}{l}1845 \\
15 \\
\end{array}$ & $\begin{array}{l}2378 \\
08 \\
\end{array}$ \\
\hline
\end{tabular}

Source: Directorate of Economics \& Statistics, Bengaluru.

Table 4 Selection of Sample Farmers in Sagar Taluk

\begin{tabular}{|c|c|c|c|c|c|c|c|c|c|c|}
\hline & stag & & First & & ond $\mathrm{s}$ & & Thirc & tage & & $\begin{array}{l}\text { Tota } \\
1\end{array}$ \\
\hline Sagar(T) & $\begin{array}{l}\text { Sm } \\
\text { all }\end{array}$ & $\begin{array}{l}\text { Mediu } \\
\mathrm{m}\end{array}$ & Large & $\begin{array}{l}\text { Sma } \\
11\end{array}$ & $\begin{array}{l}\text { Med } \\
\text { ium }\end{array}$ & $\begin{array}{l}\text { Lar } \\
\text { ge }\end{array}$ & $\begin{array}{l}\text { Smal } \\
1\end{array}$ & $\begin{array}{l}\text { Medi } \\
\text { um }\end{array}$ & $\begin{array}{l}\text { Larg } \\
\mathrm{e}\end{array}$ & \\
\hline Avinahalli & 01 & 01 & 01 & 01 & 01 & 01 & 01 & 01 & 01 & 09 \\
\hline E.J.Mane & 01 & 01 & 01 & 01 & 01 & 01 & 01 & 01 & 01 & 09 \\
\hline Total & 02 & 02 & 02 & 02 & 02 & 02 & 02 & 02 & 02 & 18 \\
\hline
\end{tabular}

Source: Field Study data 


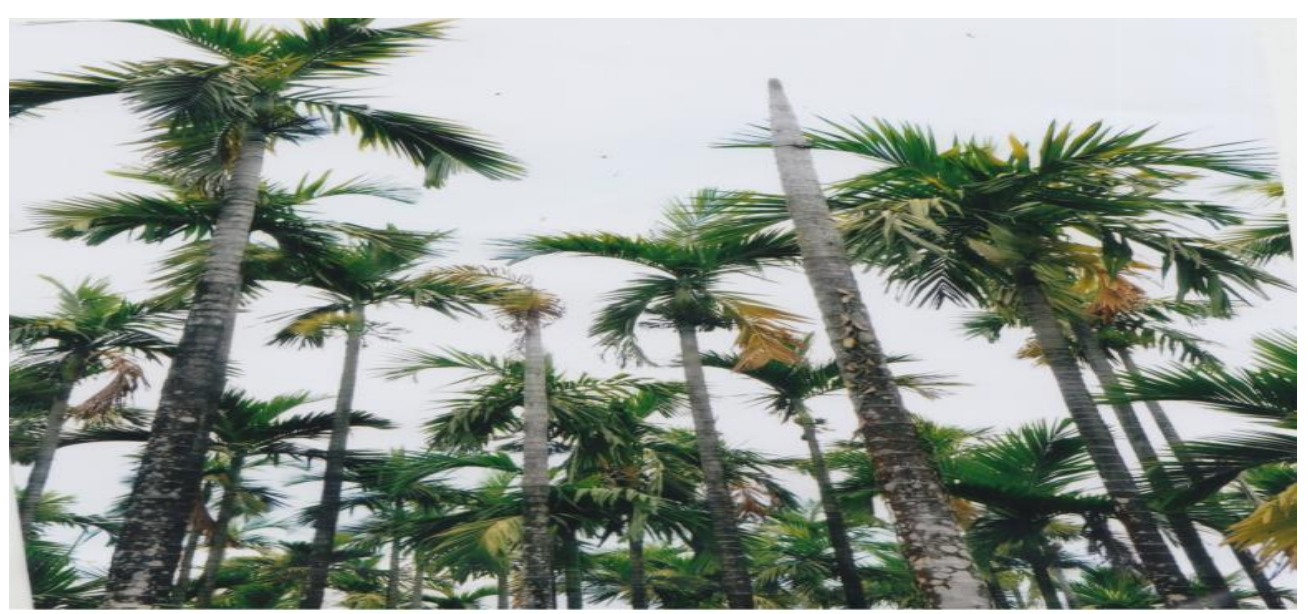

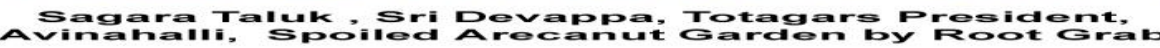

(Root Grub)

Table 5 Selection of Sample Farmers in Thirthahalli Taluk

\begin{tabular}{|l|l|l|l|l|l|l|l|l|l|l|}
\hline & \multicolumn{9}{|c|}{ First } & \multicolumn{3}{|c|}{ Second stage } & \multicolumn{3}{|l|}{ Third stage } & $\begin{array}{l}\text { Tota } \\
\text { stage }\end{array}$ \\
\hline $\begin{array}{l}\text { Thirthalli } \\
\text { (T) }\end{array}$ & $\begin{array}{l}\text { Sm } \\
\text { all }\end{array}$ & $\begin{array}{l}\text { Medi } \\
\text { um }\end{array}$ & $\begin{array}{l}\text { Larg } \\
\text { e }\end{array}$ & $\begin{array}{l}\text { Sm } \\
\text { all }\end{array}$ & $\begin{array}{l}\text { Me } \\
\text { diu } \\
\text { m }\end{array}$ & $\begin{array}{l}\text { Lar } \\
\text { ge }\end{array}$ & $\begin{array}{l}\text { Sm } \\
\text { all }\end{array}$ & $\begin{array}{l}\text { Med } \\
\text { ium }\end{array}$ & $\begin{array}{l}\text { Larg } \\
\text { e }\end{array}$ & \\
\hline Kotegadde & 01 & 01 & 01 & 01 & 01 & 01 & 01 & 01 & 01 & 09 \\
\hline Hosathota & 01 & 01 & 01 & 01 & 01 & 01 & 01 & 01 & 01 & 09 \\
\hline Total & 02 & 02 & 02 & 02 & 02 & 02 & 02 & 02 & 02 & 18 \\
\hline
\end{tabular}

Source: Field Study data

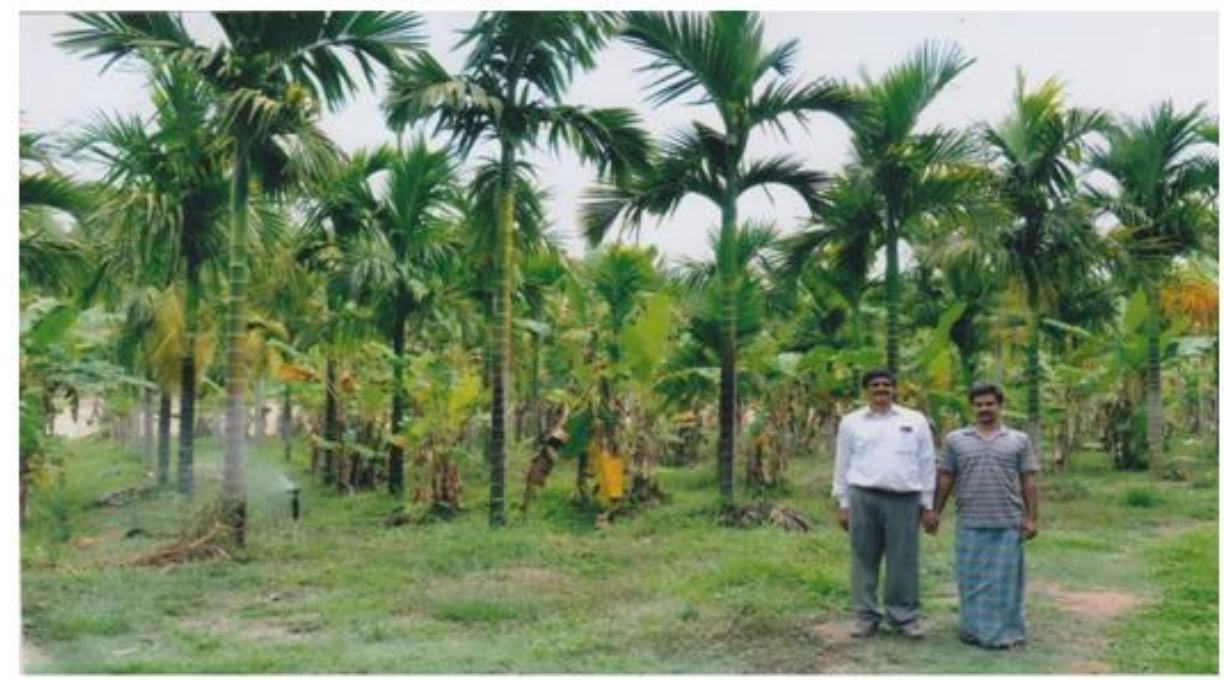

Arecanut Garden of Sri Nagarjuna and Sons, at Guddekoppa, Thirthahalli Taluk in Good Condition, Field study with Dr. B.T. Ramappa 


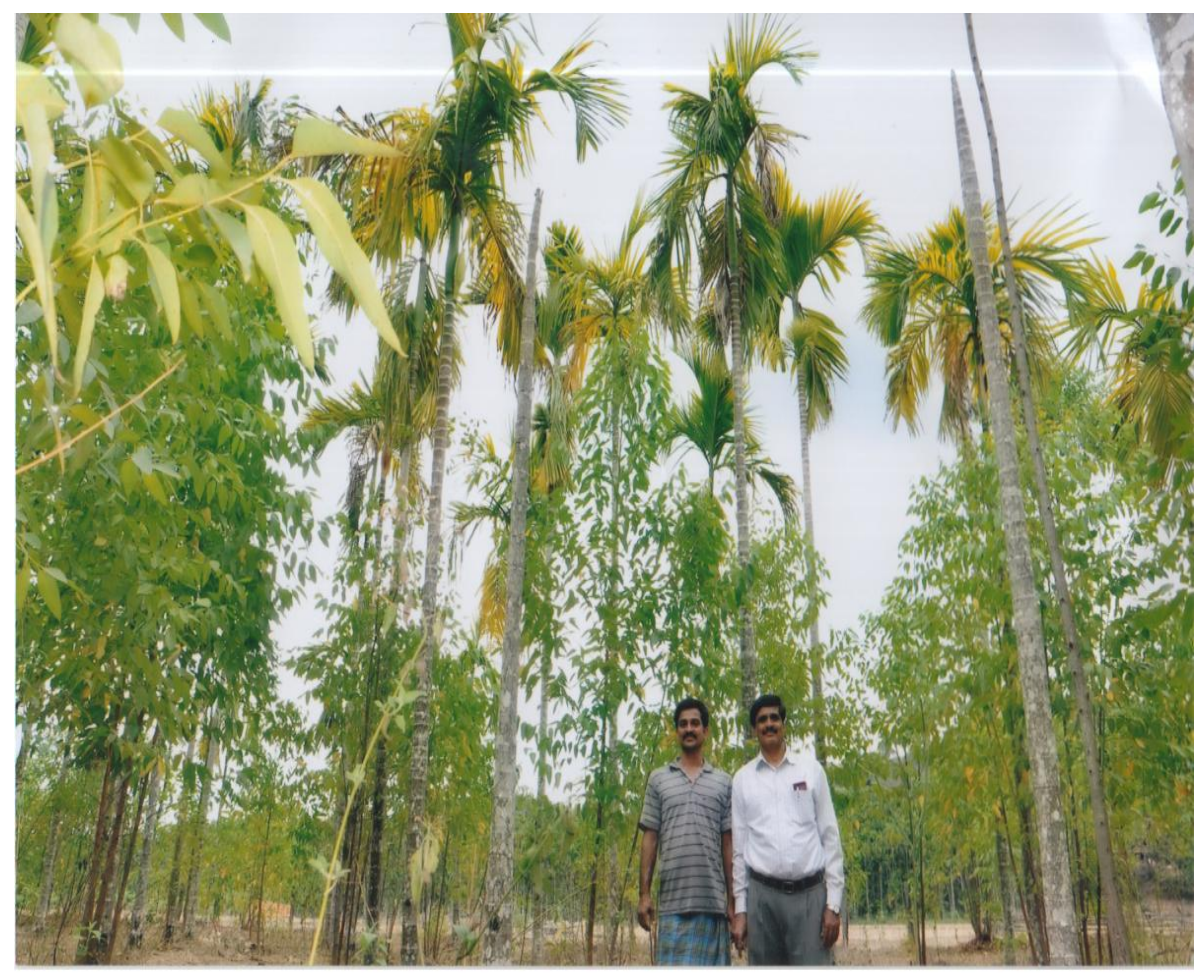

Thirthahalli Taluk, Araga Hobli, Kotegadde Nagaraj \& Sons
Spoiled Arecanut Garden, (Due to Root Grab Disease)

Table 6 Selection of Sample Farmers in Shivamogga Taluk

\begin{tabular}{|l|l|l|l|l|l|l|l|l|l|l|}
\hline & \multicolumn{9}{c|}{ First stage } & \multicolumn{3}{c|}{ Second stage } & \multicolumn{2}{|c|}{ Third stage } & Total \\
\hline Shivamogga (T) & $\begin{array}{l}\text { Sma } \\
\text { ll }\end{array}$ & $\begin{array}{l}\text { Mediu } \\
\mathrm{m}\end{array}$ & Large & $\begin{array}{l}\text { Sma } \\
\text { ll }\end{array}$ & $\begin{array}{l}\text { Medi } \\
\text { um }\end{array}$ & $\begin{array}{l}\text { Larg } \\
\mathrm{e}\end{array}$ & $\begin{array}{l}\text { Smal } \\
\text { l }\end{array}$ & $\begin{array}{l}\text { Medi } \\
\text { um }\end{array}$ & Large & \\
\hline Gajanur & 01 & 01 & 01 & 01 & 01 & 01 & 01 & 01 & 01 & 09 \\
\hline Hossalli & 01 & 01 & 01 & 01 & 01 & 01 & 01 & 01 & 01 & 09 \\
\hline Total & 02 & 02 & 02 & 02 & 02 & 02 & 02 & 02 & 02 & 18 \\
\hline
\end{tabular}

Source: Field Study data

IV.

\section{Socio-economic background of sample farmers}

A. The above sample villages are having the basic infractural facilities such as veterinary facility, primary health centre, and agro service center, primary co-operative credit society, regional rural bank (Gramina bank), Transport facilities, sub roads, main road, market, school, post \& telegraph office, Agricultural Produce Marketing Committee, Nearby Co-operative Marketing Societies (MAMCOS,CAMPCO\&Others) , Storage etc, all these facilities are available in these villages within a range of $10 \mathrm{~km}$ distance or connecting to the available facilities.

\section{B. Housing Condition of Sample Farmers}

Table 7 Housing Condition of Sample Farmers

\begin{tabular}{|c|c|c|c|c|c|c|}
\hline Farmer category & \multicolumn{2}{|c|}{ Sagar Taluk } & \multicolumn{2}{|c|}{ Thirthahalli Taluk } & \multicolumn{2}{|c|}{ Shivamogga Taluk } \\
\hline & Kutcha & Pucca & Kutcha & Pucca & Kutcha & Pucca \\
\hline Small & 85 & 15 & 88 & 12 & 83 & 17 \\
\hline Medium & 84 & 16 & 86 & 14 & 80 & 20 \\
\hline Large & 11 & 89 & 15 & 85 & 05 & 95 \\
\hline
\end{tabular}

Source;Survey Data

It is clear from the table 4 that very large number of small \& medium farmers in the study area own kutcha housesa.It indicates their poor economic condition.In case of large category, majority of them own 
pucca houses and very few will be owning kutcha houses that large farmers because of large size of land holdings earn better income \& possess better housing facilities.

The inter-taluka comparison of housing conditions of farmers reveal that in Shivamogga taluk all categories of farmers possess better housing conditions as compared to other two talukas. From this it may be concluded that majority of small \& medium farmers possess kutcha houses in the study area whereas the large farmers mainly possess pucca houses. The respective conditions are certainly better in Shivamogga taluk as compared to the other talukas.

\section{D.Family size}

Table 8 Average Family Size of Sample Farmers

\begin{tabular}{|c|c|c|c|}
\hline Farmer category & Sagar Taluk & Thirthahalli Taluk & Shivamogga Taluk \\
\hline Small & 05 & 06 & 07 \\
\hline Medium & 04 & 05 & 06 \\
\hline Large & 05 & 05 & 06 \\
\hline
\end{tabular}

Source;Survey Data

It is clear from the table 5 that the average size of family is large in case of small farmers category as compared to medium and large farmers. very large number of small \& medium farmers in the study.

\section{E. Educational Status of Sample Farmers in the Study Area.}

Table 9 Educational Status of Sample Farmers in the Study Area (percentage)

\begin{tabular}{|l|l|l|l|l|l|l|}
\hline Farmer category & \multicolumn{3}{|l|}{ Sagar Taluk (\%) } & Thirthahalli Taluk (\%) & \multicolumn{2}{l|}{ Shivamogga Taluk (\%) } \\
\hline & $\begin{array}{l}\text { Upto } \\
\text { SSLC }\end{array}$ & College & $\begin{array}{l}\text { Upto } \\
\text { SSLC }\end{array}$ & College & $\begin{array}{l}\text { Upto } \\
\text { SSLC }\end{array}$ & College \\
\hline Small & 70 & 30 & 65 & 35 & 60 & 40 \\
\hline Medium & 50 & 50 & 30 & 70 & 25 & 75 \\
\hline Large & 10 & 90 & 05 & 95 & 03 & 97 \\
\hline
\end{tabular}

Source;Survey Data

It is evident from the Table- 6 that educational status of sample farmers seems to be better in the study area. It concluded the sample farmers in the study area are educated.

\section{Educational Status of Sample Farmers in the Study Area.}

In the study area, food \& clothing account for nearly half of the total expenditure. The proportion of expenditure on education \& social items is almost same in all categories of farmers in the study area, whereas the percentage of expenditure on medicine is more in the case of small and medium farmers, \& less in case of large farmers.

Unlike field crops \& other plantation crops, arecanut has certain unique features in terms of its geographical limitations, spacing \& the type of intercrops grown with it. The average size of arecanut garden was 3.00 acres, 2.00 acres and 4.00 acres respectively in Sagar, Thirthahalli and Shivamogga. The size of the arecanut gardens was small in Thirthahalli followed by Sagar compared to other region. The number of areca palms per acre were more in case of Sagar(600),450 in Thirthahalli and 550 shivamogga taluks. The recommended spacing for the variety in the region is $9^{\prime} * 9^{*}$.

Majority of the farmers in all the regions had taken up intercrop (banana\& pepper) in their areca gardens particularly during the early stage of arecanut establishment. This has also helped the farmers in protecting the young arecanut seedlings from sunstroke. After the establishment period, farmers have taken up the intercrop (banana) in their gardens in order to utilize the space left between the areca palms. The yields of the intercrops are utilize for home consumption $\&$ the surplus is marketed.

Table 10 Arecanut cultivated area in Sagar Taluk Area Hectares

\begin{tabular}{|l|l|l|l|l|l|l|l|l|l|l|l|}
\hline Hobli wise & $\begin{array}{l}1999 \\
-00\end{array}$ & $\begin{array}{l}2000 \\
-01\end{array}$ & $\begin{array}{l}2001 \\
-02\end{array}$ & $\begin{array}{l}2002 \\
-03\end{array}$ & $\begin{array}{l}2003 \\
-04\end{array}$ & $\begin{array}{l}2004- \\
05\end{array}$ & $\begin{array}{l}2005 \\
-06\end{array}$ & $\begin{array}{l}2006 \\
-07\end{array}$ & $\begin{array}{l}2007 \\
-08\end{array}$ & $\begin{array}{l}2008 \\
-09\end{array}$ & $\begin{array}{l}2009 \\
-10\end{array}$ \\
\hline Kasaba & 615 & 649 & 649 & 746 & 746 & 804 & 823 & 808 & 808 & 808 & 915 \\
\hline Anandapur & 594 & 655 & 719 & 638 & 638 & 586 & 630 & 662 & 662 & 662 & 661 \\
\hline Avinahalli & 484 & 359 & 357 & 357 & 357 & 569 & 558 & 560 & 560 & 560 & 602 \\
\hline Karur & 409 & 419 & 512 & 512 & 512 & 526 & 522 & 539 & 539 & 539 & 650 \\
\hline Barangi & 294 & 310 & 318 & 260 & 260 & 276 & 334 & 341 & 341 & 341 & 336 \\
\hline Thalagoppa & 560 & 599 & 606 & 668 & 668 & 677 & 645 & 727 & 727 & 727 & 743 \\
\hline Total & 2956 & 2991 & 3161 & 3181 & 3181 & 3438 & 3512 & 3637 & 3637 & 3637 & 3907 \\
\hline
\end{tabular}

Source: Field study data-taluk office, Sagar 
VI.

Arecanut Prices in Shimoga \& Sagar market in Karnataka

I.1 Shimoga in the month of March 07, 2013

Bette-- Rs. 13689- Rs. 14059

Edi-- Rs. 12606- Rs. 13136

Gorabalu --Rs.6509- Rs. 9829 Rasi Edi-Rs13100- Rs. 13446

Saraku --Rs.16590- Rs. 20808(Kannada Prabha daily news )

I.2. Sagar in the month of March 07, 2013

Beligotu(White)-- Rs. 7200- Rs. 7669 Challi-- Rs. 9601- Rs. 10655

Red Gotu --Rs.8710- Rs. 9870

Rasi Edi-Rs13189- Rs. 13389

Sippegotu --Rs.4569

(Source: Kannada Prabha daily newspaper in Karnataka P.2).

VII. Varities

There are few local varities known by the name of the place where they are grown and are furnished below:-

Table 11 Verities of Arecanut in Different Area

\begin{tabular}{|l|l|}
\hline Name of the local verities & Place where grown \\
\hline South Kanara & Dakshina kannada district \& Kasaragod district of Kerala \\
\hline Thirthahalli & Malnad area of Karnataka \\
\hline Sreevardhan & Coastal Maharashtra \\
\hline Mettupalayam & Coimbatore district \\
\hline Mahitnagar & West Bengal \\
\hline Kahikuchi & Assam \\
\hline
\end{tabular}

:N.Kumar JBM Md. ABDUL KHADER et all, Introduction to Spices,Plantation Crops,Medicinal \& Aromatic Plants, OXFORD \& IBH PUBLISHING CO.PVT.LTD. New Delhi Pp. 15.02-15.03

Central Plantation Crops Research Institute, Regional Station, Vittal has released three improved cultivars, they are:

Table 12 Three Improved Cultivars

\begin{tabular}{|l|l|}
\hline Name of the Cultivar & Special attributes \\
\hline Mangala & $\begin{array}{l}\text { An introduction from China (VTL-3) early bearing, higher fruit set, higher } \\
\text { yield (10 kg ripe nuts/palm/year),semitall variety. }\end{array}$ \\
\hline Subangala & $\begin{array}{l}\text { Aselection from Indonesia 9VTL-11), yield } 17.5 \mathrm{~kg} \text { of nuts } / \text { palm at the age of } \\
10 \text { years. }\end{array}$ \\
\hline Sreemangala & A selection from Singapore (VTL-17), yield $16.5 \mathrm{~kg} / \mathrm{palm}$ at the $10^{\text {th }}$ year. \\
\hline
\end{tabular}

Source:N.Kumar JBM Md. ABDUL KHADER et all, Introduction to Spices,Plantation Crops,Medicinal \& Aromatic Plants, OXFORD \& IBH PUBLISHING CO.PVT.LTD. New Delhi Pp. 15.02-15.03

\section{PROBLEMS OF ARECA GROWERS:}

VIII. MAJOR FINDINGS OF THE STUDY:

- Yield Loss Of Arecanut In Sagar \& Thirthahalli Taluks

- The per acre yield loss of arcanut that of small,medium \& large farms due to different factors. The estimated yield loss of arecanut of small farms was found to be $100 \mathrm{kgs}$. While the loss from Koleroga was 75 percent, 7percent of yield loss was due to YLD \& the 6 percent yield loss was due to the menace of Root grub. Other factors made loss of about 12 percent. All together, due to different kinds of pests \& diseases small farms in those two taluks observed 14.98 percent economic loss.In the total yield, loss of medium farms (120 kgs) 72 percent. Of yield, loss was in the form of Koleroga, 6 percent from YLD \& 7 percent from Root grub. Other factors made 15 percent yield loss to thee total. At the aggregate level, the percent economic loss due to all kinds of estimated to 13.90. In the total yield, loss of medium farms (120 kgs) 72 percent. Of yield, loss was in the form of Koleroga, 6 percent from YLD \& 7 percent from Root grub. Other factors made 15 percent yield loss to the total. At the aggregate level, the percent economic loss due to all kinds of estimated to 13.90 .

- Kolerago:The area palm flourishes in tracts of rainfall especially in Sagar \& Thirthahalli but not so well in shivamogga taluk.

- The first visible symptom is the appearance of water soaked lesions on the surface of affected nuts. The infected nuts lose their luster. The lesions gradually spread covering the entire nuts, which rot $\&$ shed from 
the calyx. As the disease advances, the fruit stalks \& rachis of inflorescence are also affected. Affected nuts are lighter in weight, \& possess large vacuoles \& dark brown radial strands internally. Infections occurring later in the season results in drying up of nut without shedding.Afart from the quantitative loss by shedding of nuts at its various stages of development, the infected nuts are also unsuitable for chewing due to deterioration in quality. The area palm flourishes in tracts of rainfall especially in Sagar \& Thirthahalli but not so well in Shivamogga Taluk. However, it grows but the yield is less. Rain plays an important role in the initiation \& spread of the disease, since low temperature and high humidity are favorable for the growth of the fungus (Coleman, 1910). The period of the Koleroga June to September every year in the study area of Malnad (Source :primary data).

- The Yellow Leaf Disease (YLD) remains today as the most serious malady affecting the crop. The malady does not kill the palm outright but is only debilitating in nature. The disease may affect one or two leaflets in any part of the crown or the entire foliage. Tips of the chlorotic leaves eventually dry up. Tips and absorbing regions of young roots turn dark \& gradually rot. The affected fruits fall off in large numbers and at last, stage, the crown topples off leaving a base trunk.

- Anabe Roga The initial visible symptom is the yellowing of outer whorl of leaves, which gradually extends to the inner whorls. The leaves exhibit wilting symptoms and droop down covering the stem \& nuts shed. At last stage, the crown topples off leaving a base trunk.

- Bud rot affected spindle appear yellow, later changing to brown \& finally the whole spindle rots.

- Mites-Adults \& young ones suck the lower surfaces of the leaves, causing them to turn yellow \& bronzed in appearance.

- B.K.Chandrashekar KPCC Leader has urged to the Karnataka government, Yellow Leaf Disease (YLD) has been haunting areca growers in Karnataka for decades, agriculturists and researches have failed to find a solution to the problem. He said, "According to the deputy commissioner of shimoga and chickmagalur districts, as many as 410 areca growers have committed suicide in the two districts in the past decade. Frustrated with the failure of the crop, many growers have migrated because of their inability to repay debts," (Source: HINDU daily newspaper Karnataka P.4 September 29, 2011).

- Arecanut prices break a decade-old record. Mr. Bawa, who was a former member of Mangalore APMC, said the banning of sale of tobacco products in plastic sachets had adversely affected the sale of gutka, comprising both red arecanut \& tobacco.Hence; many who had the habit of consuming gutka had shifted to chewing white arecanut slices(Source: HINDU daily newspaper in Karnataka P.9 August 9, 2011).

- Lack of proper training to the farmers on aspects like grading, storage etc.

- Transportation problem.

- Non-availability of adequate organized local markets, which makes the way for the entry of intermediaries.

- Labour problems

- Uncertainty of demand for arecanut.

- Instability indices of arrivals \& prices of arecanut

- In Malnad, animals spoiled the intercrops(banana \&pepper).

- The trends in prices are found to be negative for all grades of arecanut for the present during 2011-12 \& were higher in Saraku grade followed by Bette grade.

- The trend in prices for all the grades has been ups\& downs. It may be due to the collapse of arecanut prices in the recent years.

\section{IX.Policy Implications}

- Support price needs to be revised to cover the cost of production, it protecting the farmers from loss in the arecanut production.

- Arrangements should be made to provide the new technologies to the farmers.

- Suitable steps should be initiated by the government to tackle the reasons for fall in prices of arecanut.

- Alternate use of arecanut should be looked for \& promoted.

- The import duty on arecanut was increased from $35 \%$ to $100 \%$ to safeguard the interest of the farmers by the government of India. The arecanut is brought as a dry fruit. Arecanut should not be covered under dry fruit category. Appropriate action may be instituted so that the unscrupulous import should not take place.

- President of Sringeri taluk Rita sangha demanded to the government implemented Dr. Gorakh Singh recommendation \& others demand to the leaf disease of the palm (Source: Chalagara daily local newspaper in Thirthahalli taluk P.2 January 04, 2012).

- Alternative uses of Arecanut

- The by products from nuts can be used for tanning leather. 
- $\quad$ From Husk-From fibre, for making thick board, plastics, wrapping papers can be prepared from areca pulp \& bamboo.

From leaf sheath- For making paperboards for packing purpose, Ply boards, for teachers for long distance transport, it cut downs the use of softwood timber for this purpose, used as a cheap substitute for leather in house chapples $\&$ as a cheap summer wear chappels etc.

- From Arecanut Stem \& Leaf- Nails made of areca stem widely used in furniture industry, from leaves are good source of organic manure.

In view of the numerous uses for which arecanut has been put to, it is to be assumed that it will have an impact in future also, possibly through developing suitable alternative technology for its utilization.

\section{References}

[1]. Shanmugavelu K.G.,Kumar N. 7 Peter K.V.(2012), Production Technology of Spices \& Plantation Crops, AGROBIOS (INDIA) Publication,Jodhpur-315-342

[2]. Bhandari,D.K.,(1974), Study of Inter \& Associated Crops in Areca Gardens of Malnad Tract of Karnataka, Arecanut \& Spices Bulletin,5(3):76-77.

[3]. Government of India (2003), Indian Horticultural Database, National Horticultural Board, Ministry of Agriculture.

[4]. Hazell,P.B.R., Jaramilla, M \& Williamson,A., (1990) The Relationship between World Price Instability \& Prices Farmers receive in Developing Countries. Journal of Agricultural Economics, 41(3);9-12.

[5]. Rajashekarappa, M.T., (2001), Impact of crop losses due to pests\& diseases on cost structure of arecanut cultivation. An economic analysis in traditional malnad region of Karnataka. M.Sc(Agriculture) Thesis(unpublished), University of Agriculture University Bangaluru.

[6]. Prabhakara Shetty T.K. \& Vishveshwara Gowda B.L. (2003), Areca nut Cultivation \& Its Alternative use. Published by UAS, Bengaluru, Areca nut research center, Regional Research Station, Shivamogga.

[7]. Statistics of Shimoga (2008-09), District Statistical Office Shimoga.

[8]. Arecanut (2009), Government of Karnataka Department of Horticulture, Shimoga

[9]. Ananda K.S. et.al(2000), Growth \& Yield Performance of Areca nut Verities in Malnad Region of Karnataka, Journal of Plantation Crops. Vol.28 No 2 105-109.

[10]. Shimoga district administrative appeal to the government Problems of Arecanut. (Source: Kannada prabha daily newspaper in Karnataka P.2 Januvery 2, 2012).

[11]. Acharya, S.S. \& Agarwal, N.L., (1994), Agricultural Price Analysis-Theory \& Policy.Oxpord \& IBH publishing co.pvt.Ltd.New Delhi: 293-341

[12]. Padmini, S.V. (2008), "Economics of Arecanut Cultivation in Karnataka", Serials Pub,8, xvi, P.304, New Delhi.

[13]. Sujatha and Ravi Bhat S. (2010), “Agricultural Water Management", Volume 97, Issue7,July, Directorate of Arecanut and Spices Development.Pp-988-994

[14]. M Tamilselvan, (2010), "The import of arecanuts peaked", Dr M Tamilselvan, director of Directorate of Arecanut and Spices Development. 6 July, Directorate of Arecanut and Spices Development.

[15]. Wikipedia ${ }^{\circledR},(2010)$, "Trends and problems of Arecanut" is a registered trademark of the Wikimedia Foundation, Inc., a non-profit organization.6 July Wikimedia Foundation. 